\title{
A Rare Infection Following Snakebite
}

\author{
CY Cheong, MBBS (UM), CK Lee, MS Ortho (UM), Z Zuki, MS Ortho (UM) \\ Department of Orthopaedics and Traumatology, Hospital Sungai Buloh, Kuala Lumpur, Malaysia
}

\begin{abstract}
Snakebite is very common especially in Asia. We report a rare case of Providencia rettgeri infection following snakebite on the foot. This patient was treated with early and aggressive wound debridement, daily wound dressing during hospitalization and then skin coverage with split skin graft. No anti-venom was given administered. Appropriate intravenous antibiotics were given to the patient while hospitalized and oral antibiotic were prescribed upon discharge. The outcome of this treatment was successful.
\end{abstract}

Key Words:

Providencia rettgeri, Snakebite, Wound Debridement, SplitSkin Graft

\section{CASE REPORT}

A 62-year-old lady was referred to the orthopaedic clinic with a swollen left foot after being bitten by a black snake on the same day. The type of snake was unknown to the patient. The swelling was getting worse and was associated with a bleb formation on the dorsum of the foot. Patient also developed high grade fever and diarrhea.

During admission, patient showed no evidence of envenoming including nausea, vomiting, severe abdominal pain, restlessness, ptosis, bleeding tendency, or passing out dark colour urine. Initially patient had diarrhea which subsided after a few days in ward following symptomatic treatment. Physical examination of the patient showed that the vital signs were stable. A bleb sized $6 \mathrm{~cm} \times 7 \mathrm{~cm}$ was noted on the dorsum of foot. The swollen part of the foot extended up to the mid shin. No fang marks were noted. Her cardiovascular system, respiratory system and central nervous system were normal.

The patient's vital signs were monitored closely. A circulation chart was started as well to watch out for compartment syndrome. Laboratory tests were conducted and no abnormalities were noted except for high total white cell counts. Intravenous (IV) Cloxacillin $1 \mathrm{gm} 4$ times a day (QID) and intravenous C-Penicillin 2mu QID were given during the first 2 days of admission. No anti-venom was given while patient was seen in the emergency department or during hospitalization. Three days later, the swelling on the left foot was getting worse. Wound debridement was performed and the patient was then started on IV Augmentin (Amoxicillin + Clavulanic acid) (Glaxo SmithKline) 1.2g 3 times a day. During wound debridement, extensive necrotic tissue noted. Tissue was sent for culture and sensitivity at that time. Providencia rettgeri was cultured and it was sensitive to Ampicillin, Cefuroxime, Gentamicin and Unasyn (Ampicillin + Sulbactam) (Pfizer). Hence, a two week course of IV Unasyn 1.5g TID was started.

Post-operatively, a vacuum assisted closure (VAC) dressing was applied. After 1 cycle of VAC dressing, healthy granulation tissue formed and this was followed by a split skin graft 3 days later. The patient was discharged 21 days after admission and outpatient follow-up visits were suggested.

\section{DISCUSSION}

Snakebite is a life threatening condition if not managed properly. It has been estimated that five million snakebites occur every year, resulting in about 100,000 deaths annually worldwide $^{1}$. In Malaysia alone, there are approximately 40 species of venomous snakes (18 land snakes and 22 sea snakes). Snake venom is broadly classified into hematotoxic or cardiotoxic (as in Crotalidae), neurotoxic (as in Elapidae and Hydrophiidae) or myotoxic (as in Hydrophiidae) categories. Snake venoms contain more than 20 different constituents, mainly proteins which include enzymes and polypeptide toxins.

Soft tissue infection is a major complication of snakebite with local envenoming. The proteolytic properties of snake venom cause extensive tissue destruction and devitalization, thus predisposing the wound to bacterial infection from the snake's indigenous oral flora. Atul et al found that gram positive bacteria are more common than gram negative and the most common Gram positive bacteria in venom is Staphylococcus aureus followed by coagulase negative Staphylococcus and Streptococcus spp'. Similarly, in a 12year retrospective study by Avila et al, Staphylococcus aureus was the main culprit in infection secondary to snakebite, followed by Morganella morganii and Escherichia coli. There were no cases of Providencia rettgeri infection in either study. Studies regarding oral bacterial flora of snakes or culture from snakebite wounds show that Providencia rettgeri is not a common agent ${ }^{2,3}$. 
Providencia was first isolated by Rettger in 1904. It is a urease-producing gram negative bacilli. The bacterium was initially seen in chickens in what was believed to be an epidemic of fowl cholera. The 5 species currently in the genus Providencia, in descending order of prevalence, include Providencia stuartii, Providencia rettgeri, Providencia alcalifaciens, Providencia rustigianii, and Providencia heimbachae. Almost all Providencia infections involve the urinary tract, especially in patients with chronic indwelling urinary catheters; generally these infections are sensitive to late generation cephalosporins but always resistant to tetracyclines or older penicillins.

Prophylactic antibiotics have been found to be unnecessary in treating non-venomous snakebite, as reported by Blaylock et al. ${ }^{4}$. However, the World Health Organization (WHO) recommends a prophylactic course of penicillin and a single dose of gentamicin or a course of chloramphenicol, together with a booster dose of tetanus toxoid. Extensive and early wound debridement is always indicated especially in cases where soft tissue destruction is extensive. Fujioka et al found that viper envenomation could be treated without anti-venom by performing immediate radical fang mark ablation in a report on five patients ${ }^{5}$.

Secondary bacterial infection of snakebite wound is common but Providencia rettgeri infection is quite rare. However, a good outcome is possible with early wound debridement and proper wound closure using a split skin graft, a flap or a combination of both. 


\section{REFERENCES}

1. Atul G, Sujatha S, Jaya G. Wound infections secondary to snakebite. J Infect Developing Countries. 2009; 3(3): 221-3.

2. David AW. Guidelines for the clinical management of snake bites in the South-east Asia region. World Health Organization Regional Office for New Delhi. 2005.

3. Shek K C, Tsui K L, Lam K K. Oral bacterial flora of the Chinese cobra and bamboo pit viper in Hong Kong SAR, China. $J M e d$ Hong Kong. June 2009; 15(3): 183-90.

3. Fuijioka M, Oka K, Kitamura R. Immediate radical fang mark ablation may allow treatment of Japanese viper bite without antivenom J Venom Anim Toxins incl Trop Dis. 2009; 15(1): 168-78.

4. Blaylock RS. Antibiotic use and infection in snakebite victims. South African Med J 1999; 89(8): 874-6. 